PROCEEDINGS OF THE

AMERICAN MATHEMATICAL SOCIETY

Volume 138, Number 3, March 2010, Pages 891-898

S 0002-9939(09)09912-2

Article electronically published on November 3, 2009

\title{
ON THE GLOBAL ATTRACTIVITY OF MONOTONE RANDOM DYNAMICAL SYSTEMS
}

\author{
FENG CAO AND JIFA JIANG
}

(Communicated by Jane M. Hawkins)

\begin{abstract}
Suppose that $(\theta, \varphi)$ is a monotone (order-preserving) random dynamical system (RDS for short) with state space $V$, where $V$ is a real separable Banach space with a normal solid minihedral cone $V_{+}$. It is proved that the unique equilibrium of $(\theta, \varphi)$ is globally attractive if every pull-back trajectory has compact closure in $V$.
\end{abstract}

\section{INTRODUCTION}

Since the path-breaking work of Hirsch 7 (see also 23 for details), the theory on monotone dynamical systems has been developed extensively. Hirsch [7] showed that precompact orbits of a strongly monotone dynamical system generically approach the set of equilibria. For smooth strongly monotone semiflows, the generic convergence was proved by Poláčik [18] and Smith and Thieme [24. The generic convergence to periodic orbits for strongly monotone mappings was obtained by Poláčik and Tereščák [19, 20] via the tool of exponential separation.

The generic results show that the long-run behavior for monotone dynamical systems is relatively simple no matter whether it is from the view of topology, measure or cardinality. Actually the attracting domains for its upper stable or lower stable equilibria/periodic points occupy almost the whole state space. However on the boundaries of these attracting domains, which are usually codimension one Lipschitz invariant manifolds, chaotic behavior can occur (see [3, 21, 22]), but the motions on these invariant manifolds are fairly unstable and occupy measure zero space. Therefore, during the past about 30 years, many researchers in this field tried to find some additional conditions such that all trajectories have simple longterm behavior, especially, such that every forward orbit converges to an equilibrium or fixed point (periodic solution) or almost periodic solution. In summary, there have been four types of such conditions: (1) orbital stability; (2) sublinearity; (3) the monotone systems possessing a first integral or invariant function with positive gradient; (4) the monotone systems having minimal equilibria. Most of the available convergence results require a stronger monotonicity condition. As Sontag et al. pointed out in [15, p. 297], checking this condition in practice is often not

Received by the editors September 23, 2008, and, in revised form, January 17, 2009.

2000 Mathematics Subject Classification. Primary 37H05, 37C65, 34D05.

Key words and phrases. Random dynamical systems, monotonicity, global stability.

The second author is partially supported by Chinese NNSF grants 10671143 and 10531030 and Shanghai NSF grant 09ZR1423100 and is the corresponding author.

(C)2009 American Mathematical Society Reverts to public domain 28 years from publication 
so easy, or even worse: a system may be monotone but fail to satisfy the stronger notion (see, e.g., 5, 15, 16, 25] and many references therein). The second author has introduced an integer-valued function to get rid of the irreducibility condition and proved the global convergence for various monotone systems, for example, systems for every equilibrium being stable [9], sublinearity [10, systems possessing a first integral with positive gradient 11]; this technique has even been applied to the study of skew-product monotone systems without stronger notion (see [14] and [17]). This idea was first formed by the second author in 12 to solve a global stability conjecture for three dimensional cooperative systems without irreducibility. Then he [8, 13] abstracted its essence and generally proved that on an ordered Banach space possessing lattice structure, a unique equilibrium or fixed point for monotone systems without stronger notion is globally attractive if and only if every orbit is precompact. The lattice structure assumption was later dropped by Dancer [4. This result has been frequently applied in the study of modelling biology, chemistry and control theory (see [5, 15, 16, 25] and references therein) when they lack of stronger monotonicity. The same version of result for skew-product monotone semiflow is contained in [14].

There have arisen many cooperative random or stochastic differential equations in the field of ecology, epidemiology, economics or biochemistry which generate so-called monotone random dynamical systems (RDS); see, e.g., Chueshov [2] and Arnold [1]. Chueshov [2] has founded the framework of monotone RDS, but there are very few results about long-term behavior. The purpose of the present paper is to investigate the global attractivity for monotone RDS and prove a global result for the version of [8]; that is, a unique equilibrium for a monotone RDS is globally attractive if every pull-back trajectory has compact closure in $V$.

\section{Definitions AND PRELIMINARY RESUlts}

In this section, we introduce some definitions and state some results which will be useful in subsequent sections (see, e.g., Chueshov [2] and Arnold [1]).

Let $V$ be a real separable Banach space with a closed convex cone $V_{+} \subset V$ such that $V_{+} \bigcap\left(-V_{+}\right)=\{0\}$. This cone defines a partial order relation on $V$ via $x \leq y$ if $y-x \in V_{+}$which is compatible with the vector space structure of $V$. We write $x<y$ when $x \leq y$ and $x \neq y$. If $V_{+}$has nonempty interior $\operatorname{int} V_{+}$, we say that the cone $V_{+}$is solid and $V$ is strongly ordered. We write $x \ll y$ if $y-x \in \operatorname{int} V_{+}$. We equip $V$ with the Borel $\sigma$-algebra $\mathcal{B}=\mathcal{B}(V)$ generated by open sets of $V$.

Definition 2.1 (Random dynamical system). A random dynamical system with (one-sided) time $\mathbb{T}_{+}$and state space $V$ is a pair $(\theta, \varphi)$ consisting of a metric dynamical system $\theta \equiv\left(\Omega, \mathcal{F}, \mathrm{P},\left\{\theta_{t}, t \in \mathbb{T}\right\}\right)$ and a cocycle $\varphi$ over $\theta$ of continuous mappings of $V$ with time $\mathbb{T}_{+}$, i.e., a measurable mapping

$$
\varphi: \mathbb{T}_{+} \times \Omega \times V \mapsto V,(t, \omega, x) \mapsto \varphi(t, \omega, x),
$$

such that

(i) the mapping $x \mapsto \varphi(t, \omega, x) \equiv \varphi(t, \omega) x$ is continuous for every $t \geq 0$ and $\omega \in \Omega$

(ii) the mappings $\varphi(t, \omega) \equiv \varphi(t, \omega, \cdot)$ satisfy the cocycle property:

$$
\varphi(0, \omega)=i d, \varphi(t+s, \omega)=\varphi\left(t, \theta_{s} \omega\right) \circ \varphi(s, \omega)
$$

for all $t, s \in \mathbb{T}_{+}$and $\omega \in \Omega$. 
Here a metric dynamical system $\theta \equiv\left(\Omega, \mathcal{F}, \mathrm{P},\left\{\theta_{t}, t \in \mathbb{T}\right\}\right)$ with (two-sided) time $\mathbb{T}$ is a probability space $(\Omega, \mathcal{F}, \mathrm{P})$ with a family of transformations $\left\{\theta_{t}: \Omega \mapsto \Omega, t \in \mathbb{T}\right\}$ such that

1. $\theta_{0}=i d, \theta_{t} \circ \theta_{s}=\theta_{t+s}$ for all $t, s \in \mathbb{T}$;

2. $(t, \omega) \mapsto \theta_{t} \omega$ is measurable;

3. $\mathrm{P}\left(\theta_{t} B\right)=\mathrm{P}(B)$ for all $B \in \mathcal{F}$ and all $t \in \mathbb{T}$.

Definition 2.2. An $\operatorname{RDS}(\theta, \varphi)$ is said to be order-preserving if

$$
x \leq y \text { implies } \varphi(t, \omega) x \leq \varphi(t, \omega) y \text { for all } t \geq 0 \text { and } \omega \in \Omega .
$$

Let $V$ be a metric space with a metric $\varrho$. The multifunction $\omega \mapsto D(\omega) \neq \emptyset$ is said to be a random set if the mapping $\omega \mapsto \operatorname{dist}(x, D(\omega))$ is measurable for any $x \in V$, where $\operatorname{dist}(x, B)$ is the distance in $V$ between the element $x$ and the set $B \subset V$. If $D(\omega)$ is closed for each $\omega \in \Omega$, then $D$ is called a random closed set. If $D(\omega)$ are compact sets for all $\omega \in \Omega$, then $D$ is called a random compact set. A multifunction $\omega \mapsto D(\omega)$ is said to be invariant with respect to $\operatorname{RDS}(\theta, \varphi)$ if $\varphi(t, \omega) D(\omega)=D\left(\theta_{t} \omega\right)$ for all $t>0$ and $\omega \in \Omega$.

$\mathcal{F}^{u} \equiv \bigcap_{\nu} \overline{\mathcal{F}}^{\nu}$ is said to be the universal $\sigma$-algebra associated with the measurable space $(\Omega, \mathcal{F})$, where the intersection is taken over all probability measures $\nu$ on $(\Omega, \mathcal{F})$ and $\overline{\mathcal{F}}^{\nu}$ denotes the completion of the $\sigma$-algebra $\mathcal{F}$ with respect to $\nu$. Note that $\theta_{t} \overline{\mathcal{F}}^{\nu}=\overline{\mathcal{F}}^{\nu}$ for any fixed $t \in \mathbb{T}$. Obviously, $(\mathcal{F}$ measurable $)$ random variables are $\mathcal{F}^{u}$ measurable random variables.

Let $D: \omega \mapsto D(\omega)$ be a multifunction. We call the multifunction

$$
\omega \mapsto \gamma_{D}^{t}(\omega):=\bigcup_{\tau \geq t} \varphi\left(\tau, \theta_{-\tau} \omega\right) D\left(\theta_{-\tau} \omega\right)
$$

the tail (from the moment $t$ ) of the (pull-back) trajectories emanating from $D$, and we call the multifunction

$$
\omega \mapsto \Gamma_{D}(\omega):=\bigcap_{t>0} \overline{\gamma_{D}^{t}(\omega)}=\bigcap_{t>0} \overline{\bigcup_{\tau \geq t} \varphi\left(\tau, \theta_{-\tau} \omega\right) D\left(\theta_{-\tau} \omega\right)}
$$

the (pull-back) omega-limit set of the trajectories emanating from $D$. If $D(\omega)=$ $\{v(\omega)\}$ is a single-valued function, then $\omega \mapsto \gamma_{v}(\omega) \equiv \gamma_{D}^{0}(\omega)$ is said to be the (pull-back) trajectory emanating from $v$.

The following result is adopted from [2].

Proposition 2.3. For any random closed set $\{D(\omega)\}$ the closure $\overline{\gamma_{D}^{t}(\omega)}$ of any tail $\gamma_{D}^{t}(\omega)$ is a random closed set with respect to the universal $\sigma$-algebra $\mathcal{F}^{u}$.

A random variable $u: \Omega \mapsto V$ is said to be an equilibrium of the $\operatorname{RDS}(\theta, \varphi)$ if it is invariant under $\varphi$, i.e., if $\varphi(t, \omega) u(\omega)=u\left(\theta_{t} \omega\right)$ for all $t \geq 0$ and all $\omega \in \Omega$. Let $(\theta, \varphi)$ be an order-preserving RDS. A random variable $u: \Omega \mapsto V$ is said to be

(i) a sub-equilibrium if $\varphi(t, \omega) u(\omega) \geq u\left(\theta_{t} \omega\right)$ for all $t \geq 0$ and all $\omega \in \Omega$;

(ii) a super-equilibrium if $\varphi(t, \omega) u(\omega) \leq u\left(\theta_{t} \omega\right)$ for all $t \geq 0$ and all $\omega \in \Omega$.

Let $V$ be a real Banach space. A cone $V_{+}$is said to be normal if the norm $\|\cdot\|$ in $V$ is semi-monotone; i.e., there exists a constant $c$ such that the property $0 \leq x \leq y$ implies that $\|x\| \leq c \cdot\|y\|$. It is said to be minihedral if every finite set $M$ in $V$ which is order-bounded (i.e., has an upper bound and a lower bound) has a supremum (i.e., the least upper bound) $\sup M$. 
When $V_{+}$is a solid normal minihedral cone, we have the following result, which comes from [2, p. 87] and [2, p. 90].

Theorem 2.4. Let $V_{+}$be a solid normal minihedral cone in the real Banach space $V$. Then every compact set $B \subset V$ has a supremum. Let $\{D(\omega)\}$ be a random compact set in $V$. Then $\sup D(\omega)$ and $\inf D(\omega)$ are random variables in $V$.

Remark 2.5. In Theorem 2.4, if $\{D(\omega)\}$ is a random compact set measurable w.r.t. $\mathcal{F}^{u}$, then $\sup D(\omega)$ and $\inf D(\omega)$ are $\mathcal{F}^{u}$ measurable random variables, and the proof is similar.

The following proposition shows that the pull-back omega-limit set emanating from a semi-equilibrium consists of a single equilibrium, which can be found in [2, p. 104].

Proposition 2.6. Assume $c$ is either a sub-or a super-equilibrium and assume that for any $\omega \in \Omega$ there exists $t_{0}=t_{0}(\omega)$ such that $\overline{\gamma_{c}^{t_{0}(\omega)}(\omega)}$ is a compact set in $V$. Then $\Gamma_{c}(\omega)$ consists of a single equilibrium $u$ and

$$
\lim _{t \rightarrow \infty} \varphi\left(t, \theta_{-t} \omega\right) c\left(\theta_{-t} \omega\right)=u(\omega)
$$

for all $\omega \in \Omega$ monotonically.

Remark 2.7. Note that the conclusion in Proposition 2.6 still holds if we remove the measurability from the definitions of sub-equilibrium, super-equilibrium and equilibrium.

\section{The Main Result AND ITS PROOF}

In this section we shall present our main result and give its proof. In order to do this, we need the following consequence (see, e.g., [2, p. 35]).

Proposition 3.1. $x \in \Gamma_{D}(\omega)$ if and only if there exist sequences $t_{n} \rightarrow+\infty$ and $y_{n} \in D\left(\theta_{-t_{n}} \omega\right)$ such that

$$
x=\lim _{n \rightarrow+\infty} \varphi\left(t_{n}, \theta_{-t_{n}} \omega\right) y_{n} .
$$

Now we are in a position to state our main result.

Theorem 3.2. Let $V$ be a real separable Banach space with a normal solid minihedral cone $V_{+}$. Assume that $(\theta, \varphi)$ is an order-preserving $R D S$ with state space $V$. Suppose that

(a) for any $\mathcal{F}^{u}$ measurable random variable $x(\omega), \overline{\gamma_{x}^{0}(\omega)}$ is a compact set in $V$ for each $\omega \in \Omega$, and

(b) any two $\mathcal{F}^{u}$ measurable equilibria are equal almost surely.

Then $(\theta, \varphi)$ has an $\mathcal{F}^{u}$ measurable equilibrium $w(\omega)$, and this equilibrium is globally attractive almost surely; i.e., for any random variable $x(\omega)$,

$$
\lim _{t \rightarrow+\infty} \varphi\left(t, \theta_{-t} \omega\right) x\left(\theta_{-t} \omega\right)=w(\omega) \text { for almost all } \omega \in \Omega .
$$

Proof. For any random variable $x(\omega)$, we claim that $\Gamma_{x}(\omega)$ is an invariant random compact set measurable with respect to $\mathcal{F}^{u}$.

Firstly, we prove that $\Gamma_{x}(\omega)$ is a nonempty compact set. From the definition of trajectory, we have

$$
\lim _{t \rightarrow+\infty} \operatorname{dist}\left(\varphi\left(t, \theta_{-t} \omega\right) x\left(\theta_{-t} \omega\right), \overline{\gamma_{x}^{0}(\omega)}\right)=0 .
$$


Let $t_{n} \rightarrow \infty$ and $y_{n}=x\left(\theta_{-t_{n}} \omega\right)$. Then there exists a sequence $\left\{b_{n}\right\} \subset \overline{\gamma_{x}^{0}(\omega)}$ such that

$$
\operatorname{dist}\left(\varphi\left(t_{n}, \theta_{-t_{n}} \omega\right) y_{n}, b_{n}\right) \rightarrow 0 \text { as } n \rightarrow+\infty \text {. }
$$

The compactness of $\overline{\gamma_{x}^{0}(\omega)}$ implies that for some subsequence $\left\{n_{k}\right\}$ and some $b \in$ $\overline{\gamma_{x}^{0}(\omega)}$ we have $b_{n_{k}} \rightarrow b$. This implies that

$$
\varphi\left(t_{n_{k}}, \theta_{-t_{n_{k}}} \omega\right) y_{n_{k}} \rightarrow b \in \overline{\gamma_{x}^{0}(\omega)} \text { as } k \rightarrow+\infty .
$$

Thus by Proposition 3.1 we have that $\Gamma_{x}(\omega)$ is nonempty.

Since $\Gamma_{x}(\omega) \subset \overline{\gamma_{x}^{0}(\omega)}$ and $\Gamma_{x}(\omega)$ is closed, $\Gamma_{x}(\omega)$ is a compact set.

Secondly, let us prove that $\Gamma_{x}(\omega)$ is invariant. For any $y \in \Gamma_{x}(\omega)$, using the cocycle property and Proposition 3.1, we have

$$
\varphi(t, \omega) y=\lim _{n \rightarrow \infty} \varphi(t, \omega) \circ \varphi\left(t_{n}, \theta_{-t_{n}} \omega\right) y_{n}=\lim _{n \rightarrow \infty} \varphi\left(t+t_{n}, \theta_{-t-t_{n}} \circ \theta_{t} \omega\right) y_{n}
$$

for some sequences $y_{n}=x\left(\theta_{-t_{n}} \omega\right)$ and $t_{n} \rightarrow+\infty$. Due to Proposition 3.1 this implies that $\varphi(t, \omega) y \in \Gamma_{x}\left(\theta_{t} \omega\right)$. Thus $\varphi(t, \omega) \Gamma_{x}(\omega) \subset \Gamma_{x}\left(\theta_{t} \omega\right)$ for all $t>0$ and $\omega \in \Omega$.

Assume that $y \in \Gamma_{x}\left(\theta_{t} \omega\right)$ for some $t>0$ and $\omega \in \Omega$. Proposition 3.1 implies that

$$
y=\lim _{n \rightarrow \infty} \varphi\left(t_{n}, \theta_{-t_{n}} \circ \theta_{t} \omega\right) y_{n}
$$

for some sequences $y_{n}=x\left(\theta_{-t_{n}} \circ \theta_{t} \omega\right)$ and $t_{n} \rightarrow+\infty$. The cocycle property gives that

$$
y=\lim _{n \rightarrow \infty} \varphi(t, \omega) z_{n} \text { with } z_{n}=\varphi\left(t_{n}-t, \theta_{-t_{n}+t} \omega\right) y_{n} .
$$

Since $\overline{\gamma_{x}^{0}(\omega)}$ is compact, by (3.2) there exist $\left\{n_{k}\right\}$ and $b \in \overline{\gamma_{x}^{0}(\omega)}$ such that $z_{n_{k}} \rightarrow b$ as $k \rightarrow \infty$. Moreover Proposition 3.1 implies that $b \in \Gamma_{x}(\omega)$. From (3.3) we have $y=\varphi(t, \omega) b$. Thus $\Gamma_{x}\left(\theta_{t} \omega\right) \subset \varphi(t, \omega) \Gamma_{x}(\omega)$ for all $t>0$ and $\omega \in \Omega$. Therefore $\Gamma_{x}(\omega)$ is invariant.

Finally, we show that $\Gamma_{x}(\omega)$ is a random set measurable with respect to $\mathcal{F}^{u}$. Obviously, $\Gamma_{x}(\omega)=\bigcap_{n \in \mathbb{Z}_{+}} \overline{\gamma_{x}^{n}(\omega)}$. Then since $\Gamma_{x}(\omega) \subset \overline{\gamma_{x}^{n+1}(\omega)} \subset \overline{\gamma_{x}^{n}(\omega)}$, we have

$$
\operatorname{dist}\left(y, \gamma_{x}^{n}(\omega)\right) \leq \operatorname{dist}\left(y, \gamma_{x}^{n+1}(\omega)\right) \leq \operatorname{dist}\left(y, \Gamma_{x}(\omega)\right)
$$

for any $y \in V$. Therefore we have

$$
\operatorname{dist}\left(y, \Gamma_{x}(\omega)\right) \geq \lim _{n \rightarrow \infty} \operatorname{dist}\left(y, \gamma_{x}^{n}(\omega)\right), \omega \in \Omega .
$$

Let $x_{n} \in \gamma_{x}^{n}(\omega)$ be such that

$$
\operatorname{dist}\left(y, x_{n}\right) \leq \operatorname{dist}\left(y, \gamma_{x}^{n}(\omega)\right)+\frac{1}{n}, n=1,2, \ldots
$$

Since $\gamma_{x}^{n}(\omega) \subset \overline{\gamma_{x}^{0}(\omega)}$ as $n \rightarrow \infty$ for all $\omega \in \Omega$, there exist a subsequence $n_{k}=n_{k}(\omega)$ and $b \in \overline{\gamma_{x}^{0}(\omega)}$ such that $x_{n_{k}} \rightarrow b$. By Proposition 3.1 we obtain $b \in \Gamma_{x}(\omega)$. Therefore

$$
\operatorname{dist}\left(y, \Gamma_{x}(\omega)\right) \leq \operatorname{dist}(y, b)=\lim _{k \rightarrow \infty} \operatorname{dist}\left(y, x_{n_{k}}\right) \leq \lim _{n \rightarrow \infty} \operatorname{dist}\left(y, \gamma_{x}^{n}(\omega)\right) .
$$

Thus we obtain

$$
\operatorname{dist}\left(y, \Gamma_{x}(\omega)\right)=\lim _{n \rightarrow \infty} \operatorname{dist}\left(y, \gamma_{x}^{n}(\omega)\right), \omega \in \Omega .
$$


By Proposition 2.3 $\omega \mapsto \operatorname{dist}\left(y, \gamma_{x}^{n}(\omega)\right)$ is $\mathcal{F}^{u}$ measurable for any $n$. Thus $\omega \mapsto$ $\operatorname{dist}\left(y, \Gamma_{x}(\omega)\right)$ is also $\mathcal{F}^{u}$ measurable. Hence $\Gamma_{x}(\omega)$ is a random set measurable with respect to $\mathcal{F}^{u}$.

So we have showed that $\Gamma_{x}(\omega)$ is an invariant random compact set measurable with respect to $\mathcal{F}^{u}$.

Then by Theorem 2.4 and Remark 2.5, it follows that $a(\omega):=\inf \Gamma_{x}(\omega)$ and $b(\omega):=\sup \Gamma_{x}(\omega)$ are $\mathcal{F}^{u}$ measurable variables in $V$. Since $(\theta, \varphi)$ is order-preserving,

$$
a(\omega) \leq v(\omega) \leq b(\omega) \text { for all } v(\omega) \in \Gamma_{x}(\omega)
$$

implies that

$$
\varphi(t, \omega) a(\omega) \leq \varphi(t, \omega) v(\omega) \leq \varphi(t, \omega) b(\omega) \text { for all } v(\omega) \in \Gamma_{x}(\omega) .
$$

The invariance property $\varphi(t, \omega) \Gamma_{x}(\omega)=\Gamma_{x}\left(\theta_{t} \omega\right)$ gives that

$$
\varphi\left(t, \theta_{-t} \omega\right) a\left(\theta_{-t} \omega\right) \leq v(\omega) \leq \varphi\left(t, \theta_{-t} \omega\right) b\left(\theta_{-t} \omega\right) \text { for all } v(\omega) \in \Gamma_{x}(\omega) .
$$

Since $a(\omega)=\inf \Gamma_{x}(\omega)$ and $b(\omega)=\sup \Gamma_{x}(\omega)$, we have

$$
\varphi\left(t, \theta_{-t} \omega\right) a\left(\theta_{-t} \omega\right) \leq a(\omega) \text { and } \varphi\left(t, \theta_{-t} \omega\right) b\left(\theta_{-t} \omega\right) \geq b(\omega)
$$

for all $t \geq 0$ and $\omega \in \Omega$.

Therefore, $a(\omega)=\inf \Gamma_{x}(\omega)$ and $b(\omega)=\sup \Gamma_{x}(\omega)$ are super- and sub-equilibria measurable with respect to $\mathcal{F}^{u}$, respectively. Let

$$
a_{t}(\omega):=\varphi\left(t, \theta_{-t} \omega\right) a\left(\theta_{-t} \omega\right) \text { and } b_{t}(\omega):=\varphi\left(t, \theta_{-t} \omega\right) b\left(\theta_{-t} \omega\right) .
$$

For any fixed $t>0$, the mapping $(\omega, x) \mapsto \varphi(t, \omega) x$ is $\mathcal{F} \otimes \mathcal{B}(V), \mathcal{B}(V)$ measurable (thus $\mathcal{F}^{u} \otimes \mathcal{B}(V), \mathcal{B}(V)$ measurable). Moreover, since $\omega \mapsto(\omega, a(\omega))$ is $\mathcal{F}^{u}, \mathcal{F}^{u} \otimes$ $\mathcal{B}(V)$ measurable and $\omega \mapsto \theta_{-t} \omega$ is $\mathcal{F}^{u}, \mathcal{F}^{u}$ measurable for any fixed $t \in \mathbb{T}$, it follows that $a_{t}(\omega)=\varphi\left(t, \theta_{-t} \omega\right) a\left(\theta_{-t} \omega\right)$ is measurable with respect to $\mathcal{F}^{u}$ for any fixed $t>0$. Similarly, we obtain that $b_{t}(\omega)=\varphi\left(t, \theta_{-t} \omega\right) b\left(\theta_{-t} \omega\right)$ is also measurable with respect to $\mathcal{F}^{u}$ for any fixed $t>0$. Thus by Proposition 2.6 and Remark 2.7 $\Gamma_{a}(\omega)$ and $\Gamma_{b}(\omega)$ consist of single equilibria $w_{a}(\omega)$ and $w_{b}(\omega)$ measurable with respect to $\mathcal{F}^{u}$, respectively.

From (3.5) and the definition of omega-limit set we have

$$
\Gamma_{a}(\omega) \leq \Gamma_{x}(\omega) \leq \Gamma_{b}(\omega) .
$$

By (b), we obtain $w_{a}(\omega)=w_{b}(\omega)$ almost surely. Let $w_{x}(\omega)=w_{a}(\omega)$. Then we get $\Gamma_{x}(\omega)=\left\{w_{x}(\omega)\right\}$ almost surely. If $y(\omega)$ is another random variable, by the above process, we get $\Gamma_{y}(\omega)=\left\{w_{y}(\omega)\right\}$ almost surely for some $\mathcal{F}^{u}$ measurable equilibrium $w_{y}(\omega)$. Now using (b) again, we have $w_{x}(\omega)=w_{y}(\omega)$ almost surely. Let $w(\omega)=w_{x}(\omega)$. Then it follows that for any random variable $x(\omega)$,

$$
\lim _{t \rightarrow+\infty} \varphi\left(t, \theta_{-t} \omega\right) x\left(\theta_{-t} \omega\right)=w(\omega) \text { for almost all } \omega \in \Omega \text {. }
$$

Thus $(\theta, \varphi)$ has an $\mathcal{F}^{u}$ measurable equilibrium $w(\omega)$, which is globally attractive almost surely.

Remark 3.3. If we additionally assume that the tail $\gamma_{x}^{t}(\omega)$ (and so its closure) is a random set for every $t \geq 0$, then the equilibrium given by Theorem 3.2 is measurable with respect to $\mathcal{F}$; especially for a discrete $\operatorname{RDS}(\mathbb{T}=\mathbb{Z})$ it is true. Indeed, in this case we have that $\Gamma_{x}(\omega)$ is a random set by (3.4); thus $a(\omega), b(\omega)$ and $w(\omega)$ are $\mathcal{F}$ measurable random variables. 
Remark 3.4. Chueshov in [2] considered sublinear order-preserving RDS $(\theta, \varphi)$ with the property $\varphi(t, \omega) V_{+} \subset V_{+}$for all $t>0$ and $\omega \in \Omega$. Recall that the equivalence classes under the equivalence relation defined by $x \sim y$ if there exists $\alpha>0$ such that $\alpha^{-1} x \leq y \leq \alpha x$ on the cone $V_{+}$are called the parts of $V_{+}$. Clearly $\operatorname{int} V_{+}$is a part. If a sublinear order-preserving $\operatorname{RDS}(\theta, \varphi)$ is strongly sublinear on $\operatorname{int} V_{+}$(thus $\varphi(t, \omega) \operatorname{int} V_{+} \subset \operatorname{int} V_{+}$for all $t>0$ and $\left.\omega \in \Omega\right)$, then [2, Theorem 4.2.1] implies that any two $\mathcal{F}^{u}$ measurable equilibria in $\operatorname{int} V_{+}$are equal almost surely (note that the conclusion in this theorem is also valid for $\mathcal{F}^{u}$ measurable equilibria by a similar proof). Therefore, if for any $\mathcal{F}^{u}$ measurable random variable $x(\omega) \operatorname{in} \operatorname{int} V_{+}, \overline{\gamma_{x}^{0}(\omega)}$ is a compact set in int $V_{+}$for each $\omega \in \Omega$, then by our Theorem 3.2, $(\theta, \varphi)$ has an $\mathcal{F}^{u}$ measurable equilibrium in $\operatorname{int} V_{+}$and this equilibrium is globally attractive almost surely in $\operatorname{int} V_{+}$.

Remark 3.5. For the random parabolic equations of Fisher type discussed in [6],

$$
\left\{\begin{array}{l}
u_{t}=\Delta u+m\left(\theta_{t} \omega, x, u\right) u(1-u), x \in D \\
\frac{\partial u}{\partial n}=0, x \in \partial D
\end{array}\right.
$$

where $D \subset \mathbb{R}^{N}$ is an open, bounded and connected domain with smooth boundary, $m(\omega, x, u)$ is measurable, and $m(\omega, x, u) u(1-u)$ is of Fisher type (see [6] for details). If we further assume that $m_{u}^{\prime}(\omega, x, u)<0$ for $u \in[0,1]$, then the strongly monotone RDS (see (2.2) in [6]) generated by (3.6) is strictly sublinear on $X_{+}$, where $X$ is the state space of the RDS. We claim that the RDS is strongly sublinear on $X_{+}$; indeed, given $\lambda \in(0,1)$ and $\phi \in \operatorname{int} X_{+}$, the strict sublinearity implies that $\varphi(t, \omega, \lambda \phi)>\lambda \varphi(t, \omega, \phi)$ for all $t>0$. Obviously, $\varphi(t, \omega, \phi) \in \operatorname{int} X_{+}$. By the strong monotonicity and strict sublinearity, it follows that for all $t>0$ and $s>0$

$$
\varphi\left(s, \theta_{t} \omega, \varphi(t, \omega, \lambda \phi)\right) \gg \varphi\left(s, \theta_{t} \omega, \lambda \varphi(t, \omega, \phi)\right)>\lambda \varphi\left(s, \theta_{t} \omega, \varphi(t, \omega, \phi)\right) .
$$

Thus we get $\varphi(t+s, \omega, \lambda \phi) \gg \lambda \varphi(t+s, \omega, \phi)$ for all $t>0$ and $s>0$. So if for any strictly positive $\mathcal{F}^{u}$ measurable random variable $\phi(\omega), \overline{\gamma_{\phi}^{0}(\omega)}$ is a compact set in $\operatorname{int} X_{+}$, then from Remark 3.4 we obtain that the equilibrium 1 is globally attractive almost surely in int $X_{+}$. This is just the case (2) in [6, Theorem 4.1].

\section{REFERENCES}

1. L. Arnold, Random dynamical systems, Springer, Berlin, Heidelberg, New York, 1998. MR:1723992 (2000m:37087)

2. I. Chueshov, Monotone random systems theory and applications, Lecture Notes in Mathematics, 1779, Springer, Berlin, Heidelberg, New York, 2002. MR 1902500 (2003d:37072)

3. E. N. Dancer and P. Poláčik, Realization of vector fields and dynamics of spatially homogeneous parabolic equations, Mem. Amer. Math. Soc. 140, no. 668 (1999). MR1618487 (99m:35125)

4. E. N. Dancer, Some remarks on a boundedness assumption for monotone dynamical systems, Proc. Amer. Math. Soc. 126, no. 3 (1998), 801-807. MR1443378 (98e:47085)

5. G. A. Enciso and E. D. Sontag, Global attractivity, I/O monotone small-gain theorems, and biological delay systems, Discrete and Continuous Dynamical Systems 14 (2006), 549-578. MR.2171727 (2006g:93128)

6. G. Hetzer, W. Shen and S. Zhu, Asymptotic behavior of positive solutions of random and stochastic parabolic equations of Fisher and Kolmogorov types, J. Dynam. Differential Equations 14, no. 1 (2002), 139-188. MR1878647 (2003e:60140)

7. M. W. Hirsch, Stability and convergence in strongly monotone dynamical systems, J. Reine Angew. Math. 383 (1988), 1-53. MR921986 (89c:58108)

8. J. Jiang, On the global stability of cooperative systems, Bull. London Math. Soc. 26 (1994), 455-458. MR.1308362 (95i:34089) 
9. J. Jiang, On the analytic order-preserving discrete-time dynamical systems in $\mathbf{R}^{n}$ with every fixed point stable, J. London Math. Soc. (2) 53 (1996), 317-324. MR.1373063 (97h:58131)

10. J. Jiang, Sublinear discrete-time order-preserving dynamical systems, Math. Proc. Cambridge Philos. Soc. 119 (1996), 561-574. MR1357065 (96h:34090)

11. J. Jiang, Periodic monotone systems with an invariant function, SIAM J. Math. Anal. 27 (1996), 1738-1744. MR1416516 (98h:34089)

12. J. Jiang, A note on a global stability theorem of $M$. W. Hirsch, Proc. Amer. Math. Soc. 112 (1991), 803-806. MR1043411 (92b:58119)

13. J. Jiang and S.-X. Yu, Stable cycles for attractors of strongly monotone discrete-time dynamical systems, J. Math. Anal. Appl. 202 (1996), 349-362. MR.1402605 (97e:58136)

14. J. Jiang and X.-Q. Zhao, Convergence in monotone and uniformly stable skew-product semiflows with applications, J. Reine Angew. Math. 589 (2005), 21-55. MR2194677(2006k:37031)

15. P. De Leenheer, D. Angeli and E. D. Sontag, Monotone chemical reaction networks, J. Math. Chemistry 41 (2007), 295-314. MR2343862 (2009c:92041)

16. P. De Leenheer, S. A. Levin, E. D. Sontag and C. A. Klausmeier, Global stability in a chemostat with multiple nutrients, J. Math. Biol. 52 (2006), 419-438. MR.2235513 (2007d:92077)

17. S. Novo, R. Obaya and A. M. Sanz, Stability and extensibility results for abstract skew-product semiflows, J. Differential Equations 235 (2007), 623-646. MR2317498 (2008h:37018)

18. P. Poláčik, Convergence in smooth strongly monotone flows defined by semilinear parabolic equations, J. Differential Equations 79 (1989), 89-110. MR997611 (90f:58025)

19. P. Poláčik and I. Tereščák, Convergence to cycles as a typical asymptotic behavior in smooth strongly monotone discrete-time dynamical systems, Arch. Rational Mech. Anal. 116 (1992), 339-360. MR:1132766 (93b:58088)

20. P. Poláčik and I. Tereščák, Exponential separation and invariant bundles for maps in ordered Banach spaces with applications to parabolic equations, J. Dynamics and Differential Equations 5 (1993), 279-303 [see also Erratum, ibid. 6 (1994), 245-246]. MR1223450 (94d:47064), MR 1262730

21. S. Smale, On the differential equations of species in competition, J. Math. Biol. 3 (1976), 5-7. MR 0406579 (53:10366)

22. H. L. Smith, Planar competitive and cooperative difference equations, J. Diff. Eqns. Appl. 3 (1998), 335-357. MR1618123 (99a:39032)

23. H. L. Smith, Monotone dynamical systems: An introduction to the theory of competitive and cooperative systems, Mathematical Surveys and Monographs, 41, Amer. Math. Soc., Providence, RI, 1995. MR.1319817 (96c:34002)

24. H. L. Smith and H. R. Thieme, Convergence for strongly order-preserving semiflows, SIAM J. Math. Anal. 22 (1991), 1081-1101. MR.1112067 (92m:34145)

25. E. D. Sontag, Molecular systems biology and control, Eur. J. Control 11 (2005), 396-435. MR 2201569

Department of Mathematics, University of Science and Technology of China, Hefei, Anhui 230026, People's Republic of China

E-mail address: caofeng@mail.ustc.edu.cn

School of Science and Mathematics, Shanghai Normal University, Shanghai 200234,

People's Republic of China

E-mail address: jiangjf@shnu.edu.cn 